Ann. Génét. Sél. anim., I97I, 3 (2), 20I-206.

\title{
LES RELATIONS ENTRE LES INDEX LAITIERS DES PERES ET DES FILS DES RACES BOVINES F.F.P.N., MONTBELLIARDE ET NORMANDE
}

\author{
J.-C. MOCQUOT et M. POUTOUS \\ Station de Génétique quantitative et appliquée, \\ Centre national de Recherches zootechniques, I.N.R.A., \\ 78 - Jouy-en-Josas
}

\section{RÉSUMÉ}

Les relations entre les index de matière grasse (I) des pères et des fils ont été étudiées pour 77 couples de taureaux de race frisonne (F.F.P.N.), 71 de race normande et 37 de race montbéliarde. Le pourcentage d'élimination ( $\mathrm{E}=$ nombre de lactations de moins de 150 jours $/$ Nombre total de lactations) a été inclus dans l'analyse.

Les valeurs des moyennes, des variances et des cofficients de régression sont données dans le tableau 1. Par rapport à la valeur attendue, la valeur observée de la régression est de 84 p. 100 en moyenne, mais seulement de 57 p. 100 pour la race normande. Les valeurs de E et I sont négativement liées au niveau phénotypique et génétique $(r=0,54$ entre $\mathrm{E}$ et $\mathrm{I}$ du fils, $r=$ $-0,21$ entre $I$ du père et $\mathrm{E}$ du fils). Pour la race normande, la corrélation entre les index du père et du fils augmente fortement si on introduit la valeur de $E$ du fils.

Les raisons pour lesquelles I et $E$ sont liés et pour lesquelles la valeur moyenne de $E$ est différente suivant les races sont discutées.

Les résultats de cette étude devraient être confirmés sur plus de données. Cependant il apparaît très vraisemblable que l'élimination d'une génisse en début de lactation est liée à la valeur génétique du taureau père. Par conséquent, les auteurs proposent que le calcul des index soit fait sur l'ensemble des lactations connues, sans éliminer les lactations courtes de moins de 150 jours.

\section{INTRODUCTION}

L'étude des relations statistiques entre les index "lait * d'un échantillon de taureaux F.F.N.P. et les index correspondants de leur fils, nous a conduit à estimer à 78 p. 100 l'efficacité globale de la méthode actuelle de calcul des index, l'efficacité étant mesurée par le rapport des cœefficients de régression Fils /Père estimé et théorique. Dans le cas de mâles testés sur au moins 35 à 40 filles, l'efficacité était supérieure à 100 p. 100 (Mocquot J.-C., Pourous M., 1968).

Compte tenu de l'importance en sélection de cette dernière catégorie de mâles testés en insémination artificielle, il nous a paru important d'entreprendre un travail similaire pour les principales races laitières. 


\section{TABLEAU I}

Moyennes et variances des index "production totale de matière grasse" et des pourcentages de filles éliminées de ces calculs d'index, pour les taureaux père et fils de races : F.F.P.N., montbéliarde $e t$ normande.

Coefficients de corrélation ( $\mathrm{r}$ ) et de régression (b) et ces variables 2 à 2 .

\section{F.F.P.N}

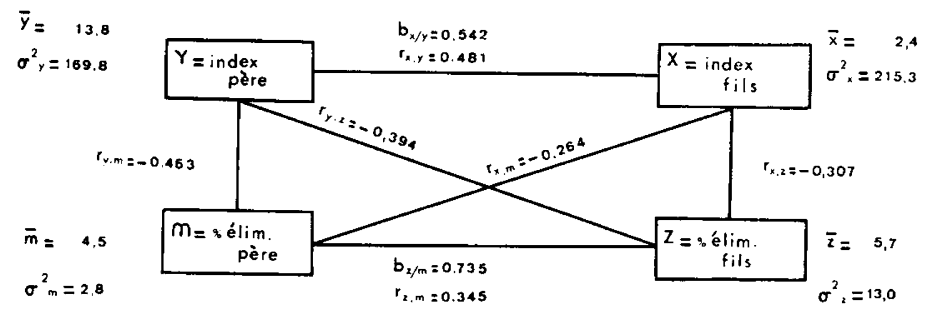

\section{MONIBEELIARDE}

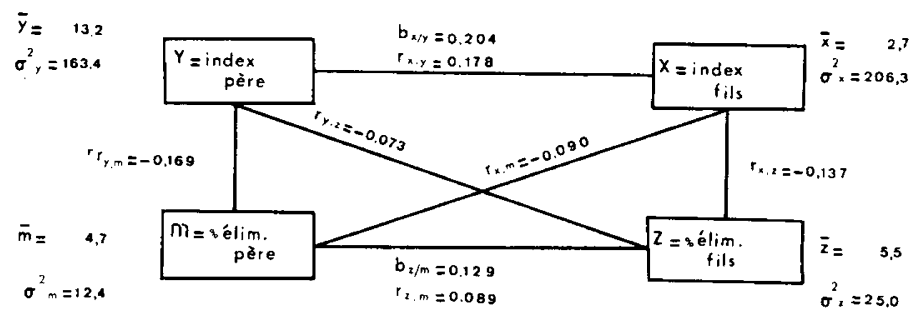

\section{NORMANDE}

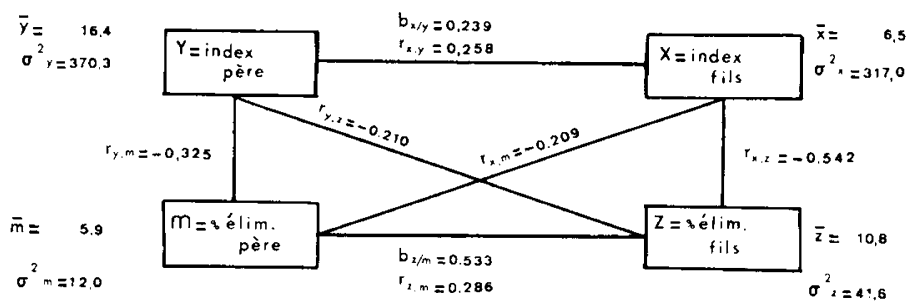

$x=$ index de matière grasse du fils,

$y=$ index de matière grasse du père,

$z=$ pourcentage d'éliminations du fils,

$m=$ pourcentage d'éliminations du père. 


\section{MATÉRIEL, MÉTHODES ET RÉSULTATS}

Le matériel comprenait les index de matière grasse de tous les couples de taureaux pèresfils figurant sur le dernier catalogue des taureaux indexés sur au moins 35 à 40 filles (C.T.C.D.C.N.R.Z., 1968), 77 couples ont été retenus pour la race F.F.P.N., 71. pour la race normande et 37 pour la race montbéliarde. On doit observer que sur les 77 couples pour la race frisonne, 51 avaient déjà été utilisés lors de notre précédente étude.
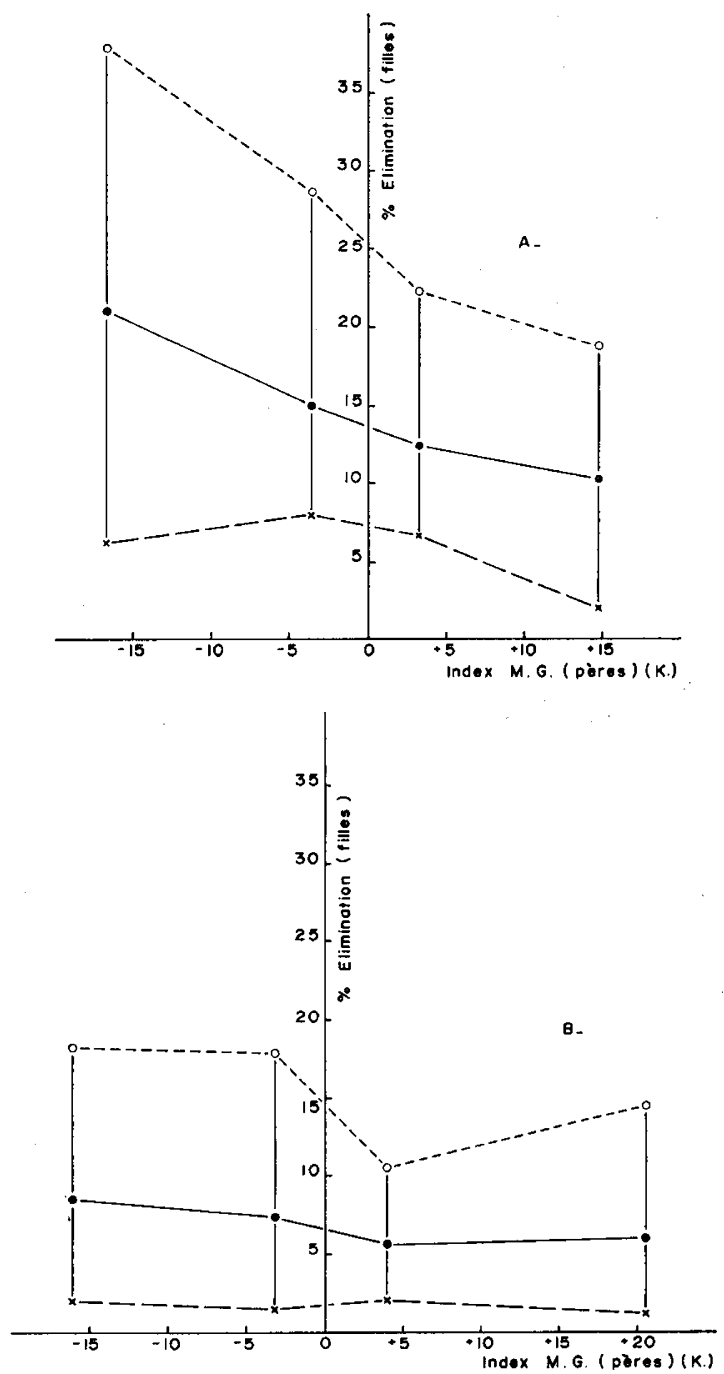

FIG, I. - Pourcentage d'éliminations (lactations inférieures à ${ }_{5} 50$ jours) en fonction de l'index de matière grasse

O . . . . O valeur la plus élevée

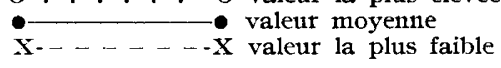

A - Race normande

B - Race F.F.P.V.

Annales de Génétique et de Sélection animale. - 1971. 
Les calculs ont porté sur les index matière grasse des pères $(y)$ et des fils $(x)$ et sur les pourcentages de lactations courtes des pères $(m)$ et des fils $(z)$. Ces derniers pourcentages sont égaux, pour un index donné, au rapport du nombre de filles ayant eu une première lactation de durée inférieure à 150 jours (performances non prises en compte pour le calcul de l'index) au nombre total de filles ayant eu une première lactation contrôlée.

A propos de l'échantillon des taureaux pères, on doit remarquer qu'il ne s'agissait pratiquement jamais de mâles mis en testage, mais en quasi totalité de reproducteurs de service pour lesquels un index a été calculé a posteriori.

Les valeurs des moyennes, des variances et des cœfficients de corrélation $(r)$ et de régression $(b)$ des variables prises 2 à 2 figurent dans le tableau 1. Dans cette étude préliminaire nous pouvons résumer les résultats comme suit :

a) L'efficacité, mesurée par le rapport, multiplié par 100, du coefficient de régression calculé de l'index du fils sur celui du père à la valeur théorique correspondante est de :

- 128 en race F.F.P.N.

- 49 en race montbéliarde.

- 57 en race normande.

- 85 pour l'ensemble des races.

b) L'importance des éliminations précoces, calculée sur l'ensemble des index publiés, est très variable suivant les races, faible pour la F.F.P.N. et la montbéliarde ( $5 \%$ ) élevée pour la normande $(\simeq 15 \%)$. On observe des variations individuelles très grandes, certains taureaux ayant jusqu'à 50 p. 100 de filles éliminées en début de lactation.

c) Par rapport à cette situation moyenne, l'échantillon des taureaux pères de l'étude apparaît comme très sélectionné dans le cas de la race normande. Par contre la variance de leur index de matière grasse est anormalement élevée.

d) Le pourcentage d'élimination est négativement lié avec l'index de l'animal correspondant. On vérifie également ce phénomène sur les figures $1 \mathrm{~A}$, où nous avons reporté les moyennes du pourcentage d'élimination et de l'index de matière grasse pour 96 reproducteurs normands testés de la Coopérative d'Élevage du Centre-Nord groupés en 4 classes d'égal effectif et 1B (96 F.F.P.N.). On remarque, par ailleurs, sur ce graphique la grande étendue de variation possible du pourcentage d'élimination même pour la classe la plus élevée où, pourtant, il atteint un minimum. Ceci est particulièrement net pour la normande.

La liaison négative ainsi mise en évidence traduit, au moins en partie, un phénomène de nature génétique. En effet les pourcentages des pères et des fils sont en corrélation positive ce qui ne serait pas le cas s'ils avaient pour seule origine l'effet de facteurs du milieu. Par ailleurs la corrélation négative entre le pourcentage d'élimination des fils et l'index du père, ou réciproquement, montre qu'il existe effectivement une liaison de nature génétique entre ces 2 variables.

e) Les corrélations partielles ou multiples entre les index de matière grasse des fils $(x)$ et des pères $(y)$ et les pourcentages d'élimination des fils $(z)$ et des pères $(m)$ figurent dans le tableau 2 .

TABLEAU 2

Corrélations partielles ou multiples entre les index matière grasse des fils $(\mathrm{x})$ et des pères (y) et les pourcentages d'élimination des fils $(\mathrm{z})$ et des pères $(\mathrm{m})$

\begin{tabular}{|c|c|c|c|c|c|c|c|c|c|c|c|c|c|c|c|c|}
\hline \multicolumn{14}{|c|}{ Race } & \multirow{2}{*}{$\begin{array}{c}F . F . P . N . \\
0,48 \mathrm{I}\end{array}$} & \multirow{2}{*}{$\begin{array}{c}\text { montbéliarde } \\
0, \mathbf{1} 78\end{array}$} & \multirow{2}{*}{$\begin{array}{c}\text { normande } \\
0,258\end{array}$} \\
\hline$r_{x \cdot y}$ & . & · & . & - & . & . & . & . & . & . & . & . & . . & & & \\
\hline$\gamma_{x \cdot y z}$ & & . & . & . & . & . & . & . & . & . & . & . & . . & 0,498 & 0,420 & 0,562 \\
\hline$\gamma_{x \cdot y m}$ & . & . & . & . & . & . & . & . & - & . & . & . & . . & $0,4^{8} 3$ & $0, \mathrm{I} 80$ & 0,290 \\
\hline$\gamma_{x, y, z}$ & . & . & . & . & . & $\cdot$ & . & . & . & . & . & . & . . & $0,4 \mathrm{I}_{4}$ & 0,163 & 0,175 \\
\hline
\end{tabular}

A la lecture de ce tableau, il apparaît que la prise en considération du pourcentage d'élimination des pères n'améliore pas sensiblement les résultats. Ceci s'explique puisque dans tous les cas la variabilité restante, après sélection des pères, est faible.

Par contre la connaissance du pourcentage d'élimination des fils augmente considérablement la précision pour les races où la variance de ce critère est forte (montbéliarde et normande).

f) En résumé, on peut dire que la connaissance des pourcentages d'élimination est d'autant plus importante à considérer que la variabilité de ce critère est plus élevée pour le lot de reproducteurs considéré. 


\section{DISCUSSION}

Cette étude met en évidence 3 points importants : pourcentage d'élimination variable suivant les races et les taureaux pères des génisses, liaison entre l'index d'un reproducteur et le pourcentage d'élimination de ses filles, enfin caractère non aléatoire de ce dernier critère.

L'existence d'une sélection massale pratiquée par les éleveurs d'après les performances de de leurs animaux est bien connue. C'est le moment et l'efficacité d'une telle sélection qui prêtent à discussion. De ce travail et de celui réalisé par l'un d'entre nous sur le taux de réforme entre la $1^{\text {re }}$ et la $2^{\mathrm{e}}$ lactation (MocQuot, ZdRAVkov, 1968) il ressort une différence nette entre les races F.F.P.N. et normande. Pour la $1^{\text {re, }}$ les éliminations sont faibles en début et élevées en fin de la $1^{\text {re }}$ lacatation, l'inverse étant vrai pour la normande. Ce phénomène a, vraisemblablement, plusieurs causes : taux de conservation des femelles plus élevé pour la frisonne, race en expansion numérique importante; taux de renouvellement du cheptel, par achats et ventes sur les marchés, traditionnellement plus fort pour les éleveurs de la race normande; enfin existence vraisemblable d'un seuil de production au vêlage en dessous duquel l'éleveur estimerait non rentable de conserver ses animaux, seuil qui serait plus fréquemment dépassé en race F.F.P.N. Il est, d'ailleurs, probable que l'éleveur prend une décision selon un ensemble de critères, en particulier par référence à un niveau fixe de production mais aussi par un classement de ses animaux entre eux d'après leurs productions contrôlées. Ce dernier point de vue semble confirmé par la liaison assez étroite observée entre les pourcentages d'élimination et d'étables où les filles du reproducteur ont produit plus que leurs contemporainse $(r=-0,55$, pour 96 mâles normands de la Coopérative d'Élevage du Centre-Nord).

La corrélation négative que l'on constate entre le pourcentage d'élimination (lactations de moins de 150 jours) et l'index calculé sur les performances des filles non éliminées en première lactation s'explique par suite d'une corrélation génétique élevée entre les premiers contrôles et la lactation totale.

Dans la descendance des taureaux de faible valeur génétique il se trouvera un pourcentage élevé d'animaux à faible production au vêlage et donc susceptibles d'être éliminés par les éleveurs. L'index de ces reproducteurs sera évidemment surestimé, puisque calculé sur un échantillon déjà trié de filles mais le biais introduit ne sera pas suffisant pour que pourcentage et index ne soient pas négativement liés. Cependant, la corrélation entre les index des pères et des fils sera diminuée puisque les différences entre les groupes de descendantes seront artificiellement diminuées alors que les différences entre les pères le seront moins du fait de la sélection à laquelle ceux-ci ont été soumis de façon directe ou indirecte sur leur pourcentage d'élimination.

\section{CONCLUSION}

Les différentes constatations faites sur nos échantillons et les explications que nous venons d'avancer nécessitent une confirmation.

Cependant, il apparaît de façon pratiquement certaine que l'élimination des filles en début de lactation est très liée à la valeur génétique du père et qu'il faudrait tenir compte de ce fait dans le calcul de l'index.

Une correction a posteriori en fonction des pourcentages d'élimination semble difficile étant donné que la moyenne des productions des filles conservées ne varie pas linéairement avec le pourcentage d'élimination.

On pourrait alors envisager de réduire encore la durée minimale afin de limiter le phénomène ou bien plus simplement de faire intervenir toutes les lactations, quelle que soit leur durée, en supposant que les causes de réforme précoce, autres que la production, sont réparties au hasard et indépendantes de la valeur du mâle. Il conviendrait alors de vérifier que l'efficacité d'un index calculée de cette façon est améliorée.

Sur un plan plus général, il apparaît important de remarquer qu'il serait souhaitable de ne pas introduire dans les schémas de calcul des hypothèses concernant les décisions à court terme que prennent les éleveurs, ces hypothèses étant basées sur des conventions qui ne correspondent pas nécessairement à la réalité.

\section{Reçu pour publication en décembre 1970.}

Annales de Génétique et de Sélection animale. - I97r. 


\title{
SUMMARY
}

\author{
RELATIONS BETWEN MILK INDEX FOR FATHERS
}

AND SONS IN F.F.P.N., MONTBELIARDE AND NORMANDE CATTIE BREEDS.

The relationships between the fat content indexes (I) of sires and sons were studied in 77 French Friesian, 71 Normandy, and 37 Montbéliarde bull couples. The elimination percentage ( $\mathrm{E}=$ number of lactations lasting less than 150 days/total number of lactations) was included in the analysis.

Means, variances and coefficients of regression are given in Table $\mathrm{I}$.

As related to the expected value, the average regression value found is 84 per cent, except in the Normandy breed, where it is only 57 per cent. The values of $E$ and $I$ are negatively related at the phenotypic and genetic level $(r=0,5$ between $\mathrm{E}$ and $\mathrm{I}$ of son; $r=0,21$ between I of sire and $\mathrm{E}$ of son). In the Normandy breed, sire-son index correlation is greatly increased if the $E$ value of the son in introduced.

The reasons for $I-E$ relation and for difference in the average value of $E$, depending on the breed, are discussed.

The results of this study should be confirmed with more data. However, it is very probable that the elimination of a heifer at the beginning of lactation is linked to the genetic value of the sire. Thus, the authors propose that calculation of the indexes be based on the whole of known lactations, without eliminating lactations shorter than 150 days.

\section{RÉFÉRENCES BIBLIOGRAPHIQUES}

ANonyme, 1968. Taureaux indexés. Races laitières 1967. Élev. Insém. (supplem. no spéc. Stat.), roo p. Mocevot J.-C., Poutous M., 1969. Relations entre les index laitiers des reproducteurs mâles pères et fils de race bovine F.F.P.N. Ann. Génét. Sél. anim., 1, 273-280.

Mocquot J.-C., Zdravkov G., I968. Note sur la longévité des vaches laitières en relation avec 1'index de leur père (non publié).

Politiek R.D., I968. Prospects of increasing the production of milk protein by breeding. Ned. Melken. Zuidveltijdschr., 22, г 79-гіг.

Poutous M., r964. Le testage des taureaux sur la production en matière azotée de leurs filles. Résultats préliminaires. Ann. Biol. anim. Biochim. Biophys., 4, 273-283.

Robertson A., OdEGARD A.K., I966. A comparison of progeny tests for milk production on fathers and sons. Acta. Agric. Scand., 17, $24 \mathrm{I}-246$.

Vos P.M., Politiek R.D., 1964. Estimation of the breeding value of a young bull on the production figures of his parents. Z. Tierzücht. Zücht Biol., 79, 310-317. 